KARL JANSEN-WINKELN

\title{
Beiträge zu den Privatinschriften der Spätzeit
}

Im folgenden sollen eine Reihe von kleineren Problemen erörtert werden, die sich bei der Lektüre spätzeitlicher Inschriften ergeben haben, vor allem in Biographien und Gebeten. Zum einen geht es um einige spezifische Wendungen, die bislang noch nicht oder unzureichend registriert und daher in der Literatur manchmal nicht erkannt worden sind, zum anderen werden Vorschläge zur Erklärung bisher mißverstandener Textpassagen gemacht.

\section{Beiträge zur Phraseologie}

A. $m h j b r$,jemandem vertrauen"

Das Verb $m h$ „füllen, voll sein“schließt dasjenige, mit dem etwas gefüllt wird bzw. von dem etwas voll ist, nach Wb II, 116-7 mit den Präpositionen $m$ (oft) und $h r$ (seltener) an. Der feststehende Ausdruck $m h$ jb „das Herz füllen (mit jemandem)“ = ,jemandem vertrauen“ wird nach Wb II, 118, 16 sogar ausschließlich mit $m$ konstruiert. In Texten der Spätzeit, von der 26. Dynastie bis zum Beginn der Römerzeit, kommt dagegen einigemal auch ganz deutlich die Formulierung $m h \mathrm{jb} r$ vor:

1. Auf dem Würfelhocker des $P 3-3 h-r \cdot f$ der Bibliothèque Nationale in Paris aus der frühen

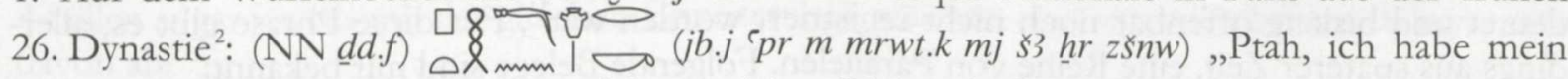
Herz mit dir gefüllt (mein Herz ist reich von deiner Liebe wie das Feld an Blumen)“.

2. Auf der Rückseite der Schreiberstatue Kairo JE 37327 des Wsr-Hnzw aus der Cachette von Karnak (etwa aus derselben Zeit) $)^{3}:(\mathrm{NN} d d . f)$ (1) (jw.j rh.kw wr.k $r$ ntrw) ,mein Herr, mein Herr, ich fülle mein Herz mit dir (denn ich weiß, daß du größer als die [anderen] Götter bist)".

3. Auf dem Sockel (untere Zeile) des Naophortorso Kairo CG 662 des T3j.f-nht aus der späten 26. oder frühen 27. Dynastie ${ }^{4}$ (NN $\left.\underline{d} d . f\right)$ (z3.tphr his.j) „O Herrin von Sais, ich habe mein Herz mit dir gefüllt ${ }^{5}$ (während dein Schutz mich umgibt)“.

4. Auf der Rückseite des Kniefigurtorso Museo Nazionale Rom 115259 aus der späten 26. bis 30. Dynastie 6 : NN [d d.ff) .... füllt (seit ich entstanden bin)“.

Abkürzungen nach Lexikon der Ägyptologie, Bd. 7, XIII-XXXVIII.

E. Ledrain, Les Monumens égyptiens de la Bibliothèque Nationale, Paris 1879, pl. LX, bestätigt durch die WB-Abschrift (WB-Mappe 183, Z. <10>), vgl. auch Kêmi 20, 1970, 46 (XIX); pl. XIII (27).

${ }^{3}$ Unpubliziert, eigene Abschrift; zur Datierung vgl. Bothmer, Egyptian Sculpture, 33.

${ }^{4}$ R. el-Sayed, BIFAO 77, 1977, 106; pl. XVII; zur Datierung s. Bothmer, Egyptian Sculpture, 70; 79.

${ }^{5}$ Das $n$ unter dem $r$ ist dem Foto nach nicht ganz sicher; vermutlich ist es nachträglich getilgt worden.

${ }^{6}$ L. Limme, Mélanges offerts à Jean Vercoutter, Paris 1985, 208. 
5. Auf der Stele London BM 379 (Z.8) des Ns-jztj aus Memphis (ptolemäisch; 3. Jahrh.) $)^{7}$ (NN

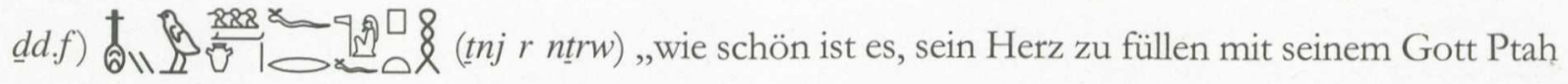
(der erhabener ist als die anderen Götter) ${ }^{68}$.

6. Auf der Stele Louvre C 316 (Z.5) des J'h-msw aus ptolemäischer Zeit (2. Jahrh.)': (PN ...

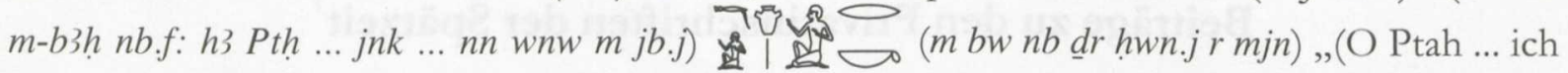
bin ... ohne Fehler in meinem Herzen,) ich habe mein Herz mit dir gefüllt (an jedem Ort seit meiner Jugend bis heute)".

7. Auf der Rückseite des Naophortorso des P3-n3-mjw der Sammlung Koradi/Berger aus dem Ende der Ptolemäerzeit ${ }^{10}$ : (NN [d dd.f Pth ...] ... jnk hmm.k ... nn jrj.n.j jht msdj.k jrj.n.j $m$ mrj jb.k m3`t

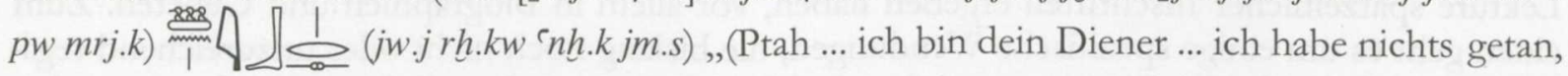
das du haßt, sondern ich handelte gemäß dem, was dein Herz liebt: das ist die Maat, die du liebst.) Ich habe mein Herz mit ihr gefüllt (denn ich weiß, daß du von ihr lebst).“

In 6 der 7 Belege kommt die Phrase nach Anrufen an einen Gott vor, meist unmittelbar nach dem Vokativ, zweimal aber auch durch weitere Sätze von ihm getrennt. Nur der 5. Beleg ist in der 3. Person gehalten, und nur in ihm steht das $r$ nicht im status pronominalis. In 5 Fällen (z. T. allerdings nur erschlossen) wird Ptah angerufen, in einem weiteren Neith; diese Belege sind also wohl aus Memphis bzw. Sais. Man könnte die Formulierung daher zunächst für eine besondere unterägyptische Variante des üblichen $m h j b m$ halten, aber immerhin stammt zumindest ein Beleg (sogar einer der ältesten, Nr. 2) unzweifelhaft aus Theben.

B. $(s) w 3 \underline{d} m n j$, den Verstorbenen gedeihen lassen, den Totenkult vollziehen“

Vor einigen Jahren war mir bei der Behandlung einer Inschrift aus der 22. Dynastie eine Wendung sw $3 \underline{d}$ mnj aufgefallen, die wörtlich soviel wie „den ,Gelandeten' gedeihen lassen“ bedeutet und bislang offenbar noch nicht registriert worden war ${ }^{11}$. Für diese Phrase gibt es, allerdings aus späterer Zeit, eine Reihe von Parallelen. Folgende Belege sind mir bekannt:

1. Auf der linken Seite des Würfelhockers Kairo JE 37527 des $\underline{D} d-B 3 s t t-j w . f-{ }^{-} n h$ aus der Cachette von Karnak (22. Dynastie): ${ }^{12}$ ( ... m3wj.j $m$ my.j $r$-h̆t hntj $m w^{-}-z 3-w^{c} n t$ prj jm.j hr-s3 [kh]kh N. Samen [noch] nach langer Zeit in ununterbrochener Generationsfolge derer, die aus mir hervorgegangen sind) als solche, die den gedeihen lassen, der gestorben ist (unaufhörlich in Karnak)“.

2. Auf der Vorderseite (Z. 14-5) des Würfelhockers Kairo JE 36918 des Hnm-jb-Re-mnw aus der Cachette von Karnak (frühptolemäisch) ${ }^{13}$ : (dd dj.frn.j $m$ jw$^{\complement} w\{. j\} n$ jw`w.j m my nt prj jm.j hn[t].sn $m$ $h n[t] . j)$ (er möge dauern lassen meinen Namen in den Erben meiner Erben, als den Samen derer, die aus mir hervorgegangen sind, indem ihr Dienst im Kult für mich besteht ${ }^{14}$ ) als solche, die den gedeihen lassen, der gestorben ist (indem sie handeln, wie ich es tat)".

E. A. E. Reymond, From the Records of a Priestly Family from Memphis, ÄA 38, 1981, 62.

Oder ,wie schön ist einer, der sein Herz füllt ...“.

E. Otto, ZÄS 81, 1956, 119-20.

${ }^{10}$ Stiftung Koradi/Berger, Zürich 1989, 72.

11 MDAIK 48, 1992, 62, Anm. 20.

${ }^{12}$ Ibid., 62-3. Leider ist in diesem Artikel irrtümlich eine falsche JE-Nummer (37597 statt 37527) angegeben worden.

${ }^{13}$ R. el-Sayed, BIFAO 84, 1984, 129.

${ }^{14}$ Oder ,,indem sie abgeordnet sind zu meinem Kult", mit passivem $s d m$, vgl. MDAIK 48, 61, Anm. 10. 
3. Auf der Vorderseite (Z. 13) des Würfelhockers Kairo JE 36579 des J'h-msw aus der Cachette

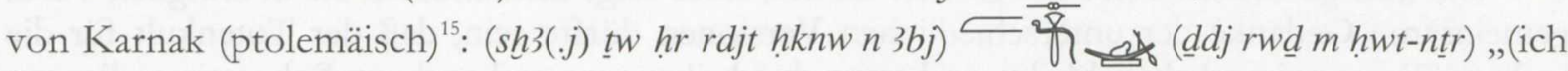
[= der Sohn] gedenke deiner [des Vaters], indem ich Lobpreis gebe unaufhörlich) als einer, der den Verstorbenen gedeihen läßt (dauernd und bleibend im Tempel)".

4. Auf der Stele (Z. 8) Wien 5857 der T3(-nt)-Dhhwtj aus Memphis (ptolemäisch) $)^{16}:(h r j w j n b m$ $p r . \underline{t} m$ h3j $h r$ sn.f) nem Grab kommt als einer, der zu seinem Bruder herabgestiegen ist) und als einer, der den Verstorbenen gedeihen läßt (solange die Götter da sind, ewig indem dein Name im Mund der Lebenden ist ...)“.

5. Auf der Rückseite (Z. 1) der Stehfigur Kairo JE 37075 des J`h-msw aus der Cachette von Karnak (ptolemäisch) ${ }^{17}:(d j . k n . j \ldots s d w h r$ r-nfr ...t t3z.n.k prt.j m-hnnw nwt.k) „(mögest du mir geben ... ein Balsamieren in schöner Weise ... nach dem du meinen ,Samen“ in deiner Stadt eingesetzt hast) als solche, die den Verstorbenen gedeihen lassen (solange die Götter da sind)“.

6. Auf der Vorderseite (Z. 6) der Gruppenstatue Kairo JE 37339 des $H r$ und des $W 3 h$-jb- $R^{c}$ aus der Cachette von Karnak (ptolemäisch; 2. Jahrh.[?]) ${ }^{18}:($ shpr.n.k pr.j mn[.tj] hrr-tp t3 hrrdw.j ḥr-s3)

降

( $m$ drw $n t r w)$, , du hast mein Haus geschaffen, indem es auf Erden bleibt und meine Kinder danach) solche sind, die den Verstorbenen gedeihen lassen (solange die Götter da sind)".

7. Auf der Rückseite (Z. 3) der Stehfigur Turin 3062 + Karnak, Karakol 258 des P3-dj-Jj-m-htp aus Theben (ptolemäisch, um 100 v. Chr. $)^{19}:(\ldots$ pr.j $m n[. t j] ~ h r d w$ nfrw $h r-s 3)$ ( $m[\underline{d} r w] n t r w),(\ldots$ indem mein Haus bleibt, und die Kinder gut sind danach) als solche, die den Verstorbenen gedeihen lassen ${ }^{20}$ ([solange] die Götter [da sind]) “.

Während es sich bei diesen 7 Beispielen deutlich um die gleiche Phrase handelt (in den letzten 4 Fällen sogar jeweils mit der gleichen Erweiterung), weichen die folgenden beiden Belege etwas davon ab:

8. Auf der Rückseite (Z. 3) des Würfelhockers Kairo JE 37149 des Wsr-Hnzw aus der Cachette von Karnak (ca. 30. Dynastie oder Ptolemäerzeit) ${ }^{21}$ heißt es: $\left(s^{\ulcorner} n h \mathrm{rn} . \mathrm{km} w \mathrm{hm}\right.$...) $J p t$-swt $n n$ skj $\underline{d} t)$,(möge dein Name [immer] aufs neue belebt werden ...) indem er dauert und frisch gehalten wird (hier in Karnak, ohne unterzugehen ewig)“. Hier ist also nur der Gebrauch von sw3 $\underline{d}$ in eben dieser Bedeutung vergleichbar.

9. Im Totenpapyrus Rhind Nr. 1 aus Theben (frührömisch) heißt es auf Seite VII, Z. 4- $5^{22}$ in

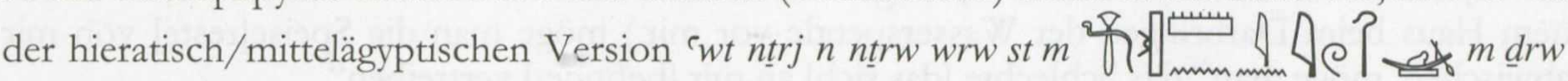
$n t r w$, die heiligen Tiere der großen Götter, sie sind gedeihend, wenn sie verstorben sind, solange

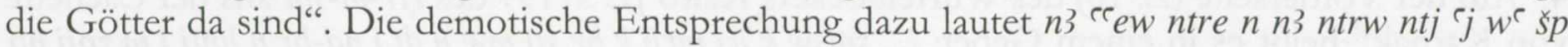

\footnotetext{
Unpubliziert, eigene Abschrift; ein Foto der Vorderseite bei R. A. Schwaller de Lubicz, Les temples de Karnak, II, Paris 1982, pl. 357.

${ }^{16}$ G. Vittmann, SAK 22, 1995, 289/293.

${ }^{17}$ Fairman, JEA 20, 1934, 2; pl. I. Zur Datierung: Fairman, op. cit., 1 und Bothmer, Egyptian Sculpture 66; 103; 128 plädieren für die Zeit Alexanders des Großen oder kurz danach (vgl. auch Übersetzungsheft zu The Temple of Khonsu, Vol. 2, OIP 103, 1981, XX, n. 26), J. Quaegebeur in: S. P. Vleeming, Hundred-Gated Thebes, Papyrologica Lugduno-Batava 27, 1995, 148-9 für die Zeit Ptolemaios’ III.

${ }_{18}$ Unpubliziert, eigene Abschrift.

19 J. Quaegebeur in: E. van t'Dack u. a., The Judean-Syrian-Egyptian Conflict of 103-101 B.C., Collectanea Hellenistica 1, 1989, 93-7.

${ }^{20} \mathrm{Vgl}$. WB I, 266, 9.

${ }^{21}$ Unpubliziert, nach eigener Abschrift.

${ }^{22}$ G. Möller, Die beiden Totenpapyrus Rhind des Museums zu Edinburg, Leipzig 1913, 34.
} 
$w^{`} \varsigma^{\ulcorner} n h h$, ,die göttlichen Tiere der großen Götter, eines folgt dem anderen bis in Ewigkeit“. Der gemeinsame Gedanke der unterschiedlichen Versionen dürfte sein, daß der Totenkult für die heiligen Tiere ewig erhalten bleibt, und zwar durch ihre ununterbrochene Sukzession, die von der demotischen Fassung hervorgehoben wird, und ebenso soll im menschlichen Totenkult die ununterbrochene Folge der Generationen das Erlöschen des Totenkults der Vorfahren verhindern (der Papyrus Rhind ist ja für diesen bestimmt, die heiligen Tiere dienen dort nur als Vergleich).

Die Phrase $(s) w 3 \underline{d}$ mnj kommt, wie gesehen, meist in sehr ähnlichen Kontexten vor. In den Belegen 1-7 ist vorher von den Nachkommen und ihrer Tätigkeit für den Vestorbenen die Rede, und das zeigt deutlich, daß $(s) w 3 \underline{d}$ mnj nur so etwas wie ,den Totenkult ausüben, aufrechterhalten" bedeuten kann, durch kultische Handlungen im engeren Sinne wie auch durch einfaches Gedenken und „Nennen des Namens“, ein Gedanke, der ja gerade in der Spätzeit besonders hervortritt ${ }^{23}$.

\section{C. $s k j \underline{d f} 3 w$ „Speise(reste) abwischen" und Vergleichbares}

1. Auf der oben (B, 1) zitierten Statue Kairo JE 37527 des $\underline{D} d-B 3 s t t-j w$ - - - $n h$ hommt auf der rechten Seite, am Ende des Textes, ein Ausdruck vor, der mir bei der Erstpublikation noch un-

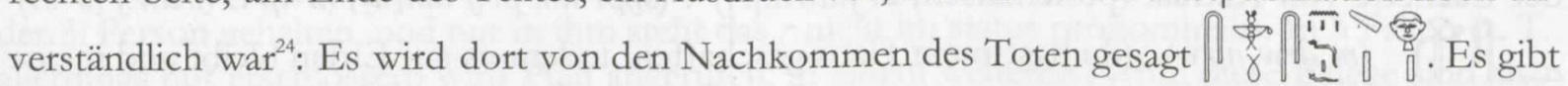
allerdings, was mir damals noch nicht bekannt war, eine ungefähre Parallele zu dieser Stelle, die sie erklären hilft:

2. Auf der Rückseite (Z. 4) des Würfelhockers Kairo JE 37354 des $\underline{D} d-h r$ aus der Cachette von

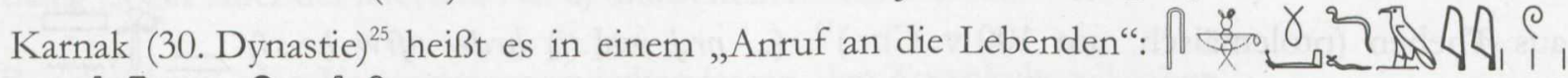

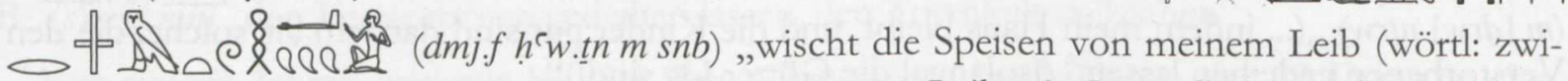
schen meinen Gliedern) ab (dann wird er [Amun] euren Leib mit Gesundheit versehen)“. Trotz des etwas merkwürdigen Determinativs | dürfte daher auch auf Kairo JE 37527 zu lesen sein „mögen sie die Speisen (oder meine Speisen bzw. Speisereste) von mir abwischen“.

Auch die folgenden Belege dürften einen vergleichbaren Sinn haben:

3. Auf der rechten Seite derselben Statue (JE 37354, Z. 13-4) wünscht sich der Tote (šzp.j snw

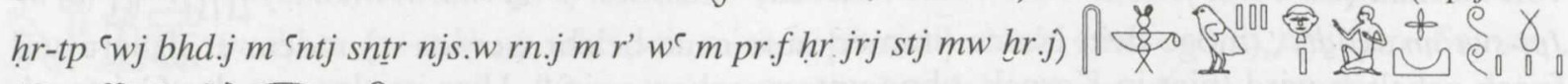

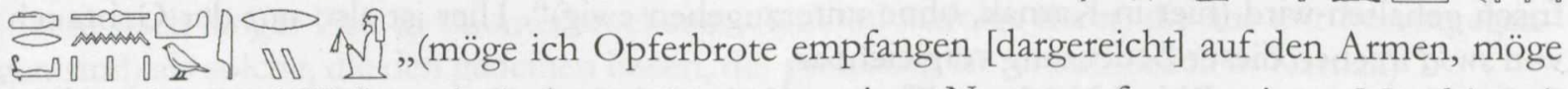
ich Myrrhen und Weihrauch riechen, möge man meinen Namen rufen aus einem Mund in seinem Haus beim Darbringen der Wasserspende vor mir,) möge man die Speise[reste] von mir abwischen, möge $\operatorname{man}^{26}$ das Schlechte [das sich] an mir [befindet] vertreiben“.

4. Auf der Vorderseite (Z. 10) des Würfelhockers Kairo JE 37199 des Hr-m-3ht aus der Cachette

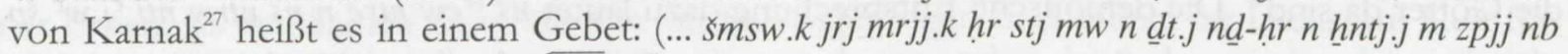
$n$ hnt.k) Wasser sprengen meinem ,Leib` [= Statue], meine Statue mit Gaben bedenken ${ }^{28}$ vom Rest deines

\footnotetext{
${ }^{23}$ Vgl. Otto, Biogr. Inschr., 64.

${ }^{24}$ MDAIK 48, 1992, 60-1, mit Anm. 11.

25 Unpubliziert, nach eigener Abschrift; zur Datierung vgl. Bothmer, Egyptian Sculpture, 100 und 103. Die recht ausführlichen Texte dieser Statue haben zahlreiche Parallelen in anderen Statueninschriften der Spätzeit.

${ }^{26}$ Zum unpersönlichen Gebrauch von .sn vgl. meine Spätmittelägyptische Grammatik, ÄUAT 34, 1996, § 219 und zur Wortstellung ibid., § 609 (mit Anm. 5).

${ }^{27}$ Unpubliziert, nach eigener Abschrift.

${ }^{28} \mathrm{~Wb}$ II, 372, 12. Sowohl $n \underline{d}-h r$ wie auch $s k$ werden noch von $h r$ abhängig sein.
} 
Opfermahls) und das Schlechte (das sich) an mir (befindet) abwischen, bestehend aus seinen [des Opfermahls] Speise[reste]n $n^{\text {c229 }}$.

5. Das Gebet auf der Vorderseite der Statue JE 36918, aus dem oben unter B,2 zitiert worden ist, schließt mit den Worten (unmittelbar im Anschluß an diese Passage) ${ }^{30}$ : (njs.w rn.j hft jrj stj

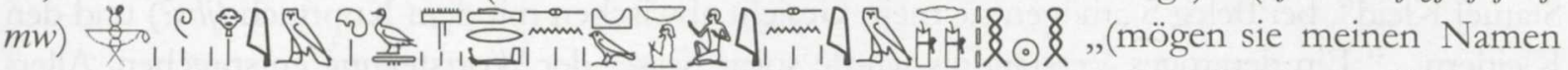
rufen, wenn eine Wasserspende dargebracht wird, ) mögen sie Verwestes(? ${ }^{31}$, Schmutz ${ }^{32}$ und Abfall(?) ${ }^{33}$ von mir abwischen, mögen sie alles Schlechte (das sich) an mir (befindet) entfernen, wenn sie dorthin kommen ( 4 statt $\mathbf{D})$, bis in alle Ewigkeit“"

6. Auf der Vorderseite des Würfelhockers Kairo 37873 des Psmtk aus der Cachette von Karnak (ca. 26. Dynastie) ${ }^{34}$ steht ein „Anruf an die Lebenden“ mit einigen ungewöhnlichen Formulierungen: (... ddd.tn n.j htp-dj-njswt ... stj n.j mw sntr $n$ k3.j) $\left.n . j n t r r r^{c} n b\right),(\ldots$ ihr sollt mir ein Königsopfer sprechen ... sprengt mir Wasser und räuchert meinem $\mathrm{Ka}$,) möget ihr den Staub ${ }^{35}$ von dieser meiner Statue ${ }^{36}$ entfernen, wischt sie mir ab von euren Opferspeisen ${ }^{37}$, preist Gott für mich täglich."

7. Auf der linken Kante der Rückenplatte des Stehfigurtorso des $H r$-nfr Lausanne 7 (frühptolemäisch) ${ }^{38}$ heißt es, wieder in einem Anruf an die Lebenden: (... tpjw t3 ... m33.sn znn pn ... dw3.tn ntr hft m33.f) $\underbrace{}_{01}$ in werden diese Statue ... möget ihr Gott für sie preisen, wenn ihr sie seht,) möget ihr den Staub von ihr entfernen beim Opfern ${ }^{39}[\ldots]$ “. Der Herausgeber übersetzt fiques“ und verweist auf Wb III, 281, 6-7, aber im Licht der anderen Belege ist es doch wahrscheinlicher, daß es sich auch hier nur um ein schlichtes Reinigen der Statue handelt, und gerade der Staub ist ja in Ägypten allgegenwärtig.

Aus diesen Passagen geht also hervor, daß auch den Tempelstatuen von „Privatleuten“ wirkliche Speiseopfer dargebracht werden (können), und man danach die Statue von eventuell herabgefallenen Resten (o ä.) zu reinigen hatte, ebenso wie man sie (vermutlich vor dem Opfer) von Staub reinigen sollte, ein ganz interessanter Aspekt des Statuenkults, der m. W. bislang noch nicht registriert worden ist.

In den Belegen 3 und 4 ist es auffällig (Hinweis G. Vittmann), daß wndw mit $\gamma$ determiniert wird, und in Beleg 5 könnte auch Determinativ zu s3t3 sein. Man könnte die entsprechenden

${ }^{29}$ Vermutlich ist $\sim \rho$ eine Verschreibung für unwahrscheinlich) wäre es allerdings, daß es sich um eine ungewöhnliche Schreibung des passiven Partizips von $s \underline{d}$ handelt („Zerbrochenes“), vielleicht im Sinne von „Brösel, Krümel“ o. ä.

${ }^{30}$ Nach eigener Kollation. Der Text bei R. el-Sayed, BIFAO 84, 1984, 129 ist nicht korrekt.

${ }^{31}$ Vgl. Wb I, 88, 15-6. Es könnten Speisereste vom letzten Opfer gemeint sein, die ja in einem heißen Land schnell verfaulen. Die Deutung ist aber, wie auch die der beiden folgenden Substantive, recht unsicher.

${ }^{32}$ Vermutlich eine Schreibung für $s 3 t$ (Wb IV, 27, 8-11); möglich, aber wohl weniger wahrscheinlich, wäre auch z3tw „Erde“ (im Sinne von „Schmutz“, vgl. Wb III, 424, 1).

${ }^{33}$ "Sehr unsicher, vielleicht $k f j$ zu lesen, als (gerundivisches) passives Partizip („zu Entfernendes“). Denkbar wäre aber auch $h 3 j t$,Schmutz“ (Wb III, 15, 14-6), mit dem Determinativ von $h 3 j$,nackt sein“ als Ideogramm.

${ }^{34}$ Unpubliziert, nach eigener Abschrift. Die Texte dieser Statue enthalten eine ganze Reihe von determinativlosen und ,alphabetischen" Schreibungen, die ja in dieser Zeit nicht ungewöhnlich sind.

${ }^{35}$ Vgl. auch unten, Beleg 7.

$h r$ znn.jpn; 3 als Lautzeichen für $n n$.

${ }^{37} s k k n(. j) s(w) m s n w . t n$. Die Form $s k k$ ist $\mathrm{m}$. W. einmalig. Vermutlich vertritt sie einen Imperativ mit $j$-Augment (j.sk). Der Ersatz von anlautendem $j$ durch Reduplikation des zweiten starken Radikals ist auch sonst einigemal belegt. Ich werde an anderer Stelle darauf eingehen.

${ }^{38}$ H. Wild, BIFAO 54, 1954, pl. I/III; 206-8.

39 $m d r[p \ldots]$. 
Worte daher auch als Schreibungen von sd „Kleid“ betrachten (vgl. auch die Schreibungen bei Janssen, Prices, 272ff.). Dann wäre bei Beleg 3 „,möge man mein Gesicht < mit $>$ den Kleidern abwischen“ (о. ̈.) zu verstehen, bei Beleg $4, \ldots$ und das Schlechte an mir abwischen mit ihrem [der Statue] Kleid“, bei Beleg 5 ,mögen sie mein Gesicht abwischen mit dem Kopftuch (jbs?) und den Kleidern ..." Ein derartiges Verständnis würde sogar besser der Wortstellung entsprechen. Allerdings erscheint es mir äußerst zweifelhaft, daß Privatstatuen ,bekleidet“ gewesen sein sollten.

\section{D. twt $n . j$, ,es gebührt mir, es kommt mir zu“"}

Im Unterschied zu den zuvor behandelten Wendungen ist diese im Wörterbuch dokumentiert $(\mathrm{V}, 257,9)$, allerdings geht aus den dort zitierten zwei Belegen nicht hervor, daß sie typischerweise in einem bestimmten Kontext auftritt. Da sie außerdem auch schon einigemal mißverstanden worden ist, kann auch hier eine Aufstellung der Belege vielleicht von Nutzen sein.

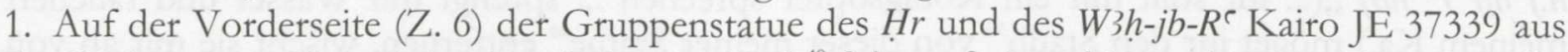
der Cachette von Karnak (ptolemäisch, s. o., B, 6) ${ }^{40}$ folgt auf einen biographischen Text über die

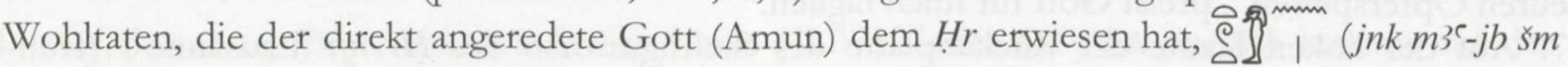
hr mw ntr.f) ,es gebührt mir; (ich war einer mit gerechtem Herzen, der seinem Gott ergeben ist) ".

2. Auf der Rückseite (Z. 4) der Stehfigur des Hr-k3wj Kairo JE 37328 aus der Cachette von

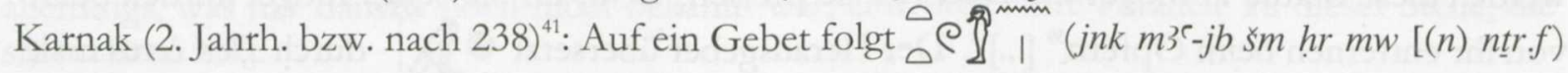
„es gebührt mir (ich war einer mit gerechtem Herzen, der [seinem Gott] ergeben ist) “.

3. Auf der Rückseite (Z.3) der Stehfigur Turin $3062+$ Karnak, Karakol 258 aus Theben

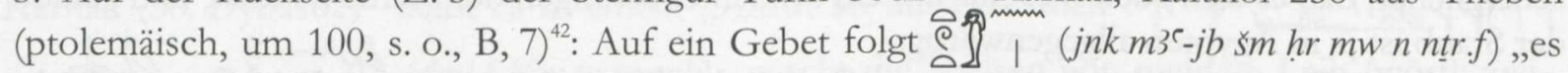
gebührt mir; (ich war einer mit gerechtem Herzen, der seinem Gott ergeben ist)“.

4. Auf der linken Seite (Z. 12) der Stehfigur des J`h-msw Kairo JE 37075 aus der Cachette von Karnak (frühptolemäisch, s. o., B, 5) ${ }^{43}$ : Nach einem Anruf an die Lebenden mit folgender Sen-

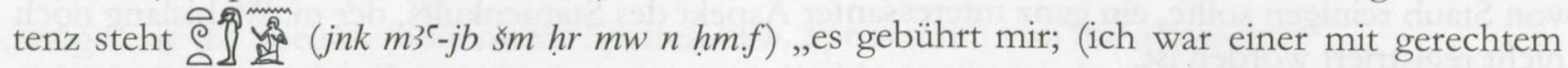
Herzen, der seiner Majestät [= Amun] ergeben ist) “.

5. In Z. 14 der Totenstele der T3-hbt aus Achmim (ptolemäisch) ${ }^{44}$ folgt auf einen Anruf an die

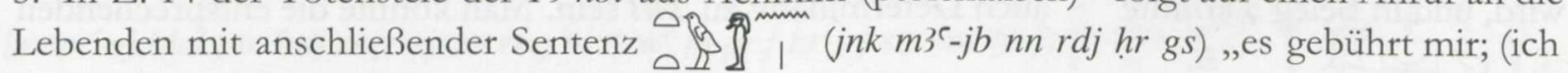
war eine mit gerechtem Herzen, ohne parteiisch zu sein)“.

6. Auf der Rückseite (Z. 3) eines weiblichen Stehfigurtorso im Museum Simu in Bukarest (ptolemäisch) ${ }^{45}$ : Auf einen Anruf an die Lebenden folgt o (jnk smsj ntr jrjw n.tn mjtt jn jwjw hr-s3) , ,es gebührt mir; (ich war eine, die Gott diente; möge das gleiche für euch getan werden durch die später Kommenden)“.

7. Auf der Rückseite (Z. 3) der knienden Altarträgerstatue des P3-dj-m3j-hss3 Leiden AST 71 aus Tell el-Moqdam (30. Dynastie oder später) ${ }^{46}$ : Auf einen Anruf an die Lebenden folgt ê]ฺ (jnk šmsj ntrr-nwtj.f) ,es gebührt mir; (ich war einer, der seinem Stadtgott diente)“.

\footnotetext{
${ }^{40}$ Unpubliziert; eigene Abschrift.

${ }^{41}$ Unpubliziert; eigene Abschrift.

${ }^{42} \mathrm{~J}$. Quaegebeur in: Van t'Dack, The Judean-Syrian-Egyptian Conflict, Collectanea Hellenistica I, Brüssel 1989, 93-7; 101 (30).

${ }^{43}$ H. W. Fairman, JEA 20, 1934, 4; pl. I (Fairman hat die Stelle allerdings mißverstanden).

${ }^{44}$ E. A. W. Budge, Some Account of the Collection of Egyptian Antiquities in the Possession of Lady Meux, ${ }^{2}$ London 1896, 132.

${ }^{45}$ G. Roeder in: Fs Griffiths, 333.

${ }^{46}$ K. Jansen-Winkeln, OMRO 77, 1997, 88.
} 
8. Auf der Rückseite (Z. 3) des Würfelhockers Kairo JE 47277 des Ns-Mnw aus Theben (früh-

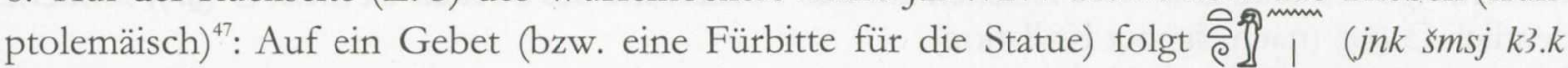
$j 3 m$-jb $n n b . f m^{\circ}$ ) , „es gebührt mir; (ich war einer, der deinem $\mathrm{Ka}$ diente, der wirklich freundlich war zu seinem Herrn)“.

9. Auf der rechten Seite (Z. 7) derselben Statue ${ }^{48}$ : Auf einen Anruf an die Lebenden folgt 命] (jnk j3m-jb jrj.n.j $3 w n{ }^{\ulcorner} h{ }^{\ulcorner} w(. j) ~ h r d w 3$ ntr $n$ znnw nb sw3w.n.j hr.sn), „es gebührt mir; (ich war einer mit freundlichem Herzen; ich habe mein ganzes Leben damit verbracht, Gott zu preisen für alle Statuen, an denen ich vorbeigekommen bin)".

10. Alle bisher angeführten Belege stammen aus Privatinschriften, überwiegend von Statuen. Der folgende Beleg (einer der beiden im Wb zitierten Fälle) stammt aus einem Text auf dem Propylon des Monthtempels in Karnak, aus der Zeit Ptolemäus' III. und IV. ${ }^{49}$ Er ist damit ein Zeugnis für die sich in späten Tempeltexten deutlich abzeichnende Tendenz, die Phraseologie der Privatinschriften (v. a. der Biographien) in den königlichen Bereich zu übernehmen ${ }^{50}$. In einer Rede des Königs an den Gott heißt es: (.. hrp.j n.k r'w nb .. jw.w n k3.k Hrz3 $3 s t z 3$ Wsjr $m$ wd

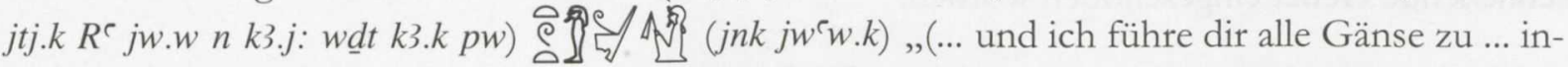
dem sie für deinen Ka bestimmt sind, Harsiese, Sohn des Osiris, auf den Befehl deines Vaters $\mathrm{Re}$, und indem sie für meinen $\mathrm{Ka}$ bestimmt sind: Das ist der Befehl deines Ka.) Es gebührt mir; (ich bin dein Erbe.) ${ }^{\text {c }}$

Charakteristisch für die Phrase twt n.j ist es, daß sie den Übergang in eine andere Textsorte anzeigt. Es folgt darauf ein (meist kurzer) biographischer Text, zumindest in den Privatinschriften (1-9). In Beleg 10 kann man $j n k j w^{\ulcorner} w . k$ natürlich nicht als Biographie bezeichnen, aber der Satz ist zumindest nach deren Vorbild formuliert. Bei dem twt n.j vorausgehenden Textsegmenten handelt es sich in den meisten Fällen um Bitten, und zwar um Gebete (Belege 2-3; 8) oder Anrufe an die Lebenden, die ja eine Bitte an die Nachwelt enthalten (Belege 4-7; 9; in 4 und 5 folgt auf die Bitte noch eine kurze Sentenz). Nur Beleg 1 fällt etwas aus dem Rahmen: Nach Ausweis der in ihm gebrauchten Verbalformen (sdm.n.f) handelt es sich bei diesem Text nicht um ein Gebet, sondern um eine Biographie; sie ist allerdings zumindest in der Redekonstellation eines Gebets (der Gott wird vom Sprecher explizit angeredet) gehalten. Auch in Beleg 10 geht keine eigentliche Bitte voraus; allerdings entspricht die vom König formulierte Aussage pragmatisch durchaus einer Bitte.

\section{Corrigenda zu einigen Inschriften}

A. Vor einiger Zeit hat E. Graefe die Götterstatue London BM 1162 des Majordomus der Gottesgemahlin Schoschenk (A?) aus der 26. Dynastie publiziert ${ }^{51}$, dabei aber die Disposition der Inschrift nicht ganz richtig verstanden. Tatsächlich ist die um den Sockel laufende Zeile (Text C bei Graefe) die Fortsetzung der beiden Zeilen auf der Oberseite des Sockels („Text B“), und der Beginn der umlaufenden Zeile ist die Vorderseite des Sockels (also unmittelbar unter der zweiten Zeile von „Text B“, wie man das auch sonst häufig findet), nicht die linke Seite wie bei Graefe. Unter Beibehaltung seiner Numerierung ist die Reihenfolge also B, 1-2-C, 2-4-C, 1 . Graefe hat sich durch eine falsche Lesung einer einzigen Hieroglyphe den Blick für die wirkliche

${ }^{47}$ G. Dares sy, ASAE 22, 1922, 266.

48 Loc. cit. („à gauche“).

49 Urk VIII, 30,6 (= $\int 34$, f), vgl. auch H. Sternberg-El Hotabi, Der Propylon des Monthtempels in KarnakNord, GOF IV, 25, 1993, 116 .

${ }^{50}$ Vgl. dazu v. a. E. Otto, Gott und Mensch, Heidelberg 1964, passim.

${ }^{51}$ Untersuchungen zur Verwaltung und Geschichte der Institution der Gottesgemahlin des Amun vom Beginn des Neuen Reiches bis zur Spätzeit, ÄA 37, 1981, Bd. 1, 219-21; Taf. 8*; 16-7 (P 20). 
Reihenfolge verstellt: Das erste Zeichen auf der Vorderseite des Sockels ist nicht $\square(\underline{d} r)$, sondern deutlich $\square(\underline{h r})$ (nach eigener Kollation).

Insgesamt lautet die Inschrift dann:

„Sprechen von Worten: ,Isis, die Große, die Gottesmutter, die Herrin des Himmels und Herrscherin aller Götter, die Schutz ausübt für ihren Bruder Osiris, die seine Feinde niederwirft unter ihn, die Herrin des Lebens, Herrscherin des Schicksals und des Gedeihens, die die Neunheit durch ihren Befehl geschaffen hat, die Freude verleiht ihrem Vater Re und den vernichtet, der sich gegen ihn auflehnt ${ }^{652}$, durch ihren geliebten Diener, den Erbfürsten und Grafen, königlichen Siegler und einzigartigen Freund, den Beliebten, der den Weg seiner Herrin befolgt, den Majordomus der Gottesverehrerin Ššnq: ,Gedenke meiner, meine geliebte Herrin, ich bin dir treu gewesen; gib mir die Belohnung (dafür) in einer langen Lebenszeit und einem schönen Begräbnis am Ende der Lebenszeit(?) $)^{53}$, indem mein Name bleibt ohne zu vergehen. “ “

Die Präposition jn in Zeile „C, 3 “ bezieht sich natürlich auf das einleitende $d d-m d w$ in „,, 1 “": Die Angabe des Sprechers ist also zwischen die einleitende eulogische Anrufung und das anschließende Gebet eingeschoben worden.

B. In demselben Werk hat Graefe auch die Osirisstatue Louvre N 3952 des Schreibers der Gottesgemahlin Pth-jrj-dj-s aus der 26. Dynastie veröffentlicht ${ }^{54}$. Auch dort hat er die Lesefolge teilweise verkannt: Der Text beginnt auf der Oberseite des Sockels mit 2 Kolumnen und 4 Zeilen, setzt sich dann auf dessen Seitenflächen fort, und zwar zunächst auf der Vorderseite (Z. 7, unterhalb der letzten Zeile auf der Oberseite), dann auf der rechten Seite (Z. 8), ganz entsprechend der Leserichtung der Hieroglyphen. Dann aber soll der Text unvermittelt auf die rechte Seitenwand des Rückenpfeilers überspringen (Z. 9), sich auf der linken fortsetzen (Z. 10), um schließlich wieder auf die Seitenflächen des Sockels (Rückseite - linke Seite, Z. 10-11) unmittelbar an Z. 8 anschließend zurückzukehren. Zu dieser schon auf den ersten Blick merkwürdigen Reihenfolge ist der Herausgeber offenbar gelangt, weil er die Gruppe $\frac{\infty}{\frac{\pi}{\Delta}}$ in Z. 8 als jrt- $H r$ auffaßt und auf die Statue selbst (als Gegenstand eines Stiftungsaktes) bezieht. Das wäre aber eine Formulierung, die in Texten dieser Art m. W. ganz einmalig wäre. Ebenso erwartet man nach der einleitenden Hymne keineswegs eine „Stiftungsformel“, sondern eine Bitte, also ein Gebet oder eine Fürbitte, und darum dürfte es sich hier in der Tat auch handeln, beginnend in der Lücke in der Mitte von Z. 8. Und dann ergibt sich als Lesefolge auch genau das, was man unbefangenerweise erwarten sollte, nämlich die Inschrift auf der Oberseite des Sockels, dann die rund um den Sockel und schließlich die linke und rechte Seitenwand des Rückenpfeilers. Unter Beibehaltung der Zeilennumerierung des Herausgebers sieht die Reihenfolge also folgendermaßen aus: Z. $1-8,10-12,9$.

Die Fürbitte verstehe ich folgendermaßen:

„[Komm $]^{55}$ in Frieden zu deinem [Diener $]^{56}$, der deinen Weg (frei)macht ${ }^{57}$, dem Sohn von Ehrwürdigen $^{58}$, die dir ergeben waren, der Schreiber der Gottesverehrerin Pth-jrj-dj-s, der Sohn des Schreibers und Kammerherrn der Gottesverehrerin Bn-3tj-dw3t-ntr , des Sohnes des Schreibers

${ }^{52}$ Zweifellos so zu verstehen, vgl. auch G. Vittmann, WZKM 75, 1983, 203.

${ }^{53} m$ ph(wj) $r$ ? Sehr unsicher und nur möglich, falls $r r$,Zeit" wie $t r$ im Sinne von „Lebenszeit“ gebraucht werden kann.

${ }^{54}$ Op. cit., 239-41; Taf. 16*-18*; 30-31 (P 38).

${ }^{55}$ Wenn - wie anzunehmen - Graefes Ergänzungsvorschlag [`wj.f] hinter wț $n h \underline{h}$ $m$ richtig ist (s. u.), bleibt gerade noch genügend Platz für [

${ }^{56} n[h m] . k$ o. ä.

${ }^{57} \mathrm{Zu}$ jrj $w 3 t$ vgl. Wb I, 247, 7-8 und die Angaben bei Meeks, Année lexicographique, 77.0808; 78.0853; 79.0585. Hier wird damit wohl einfach das Aufstellen der Götterstatue gemeint sein.

${ }_{58}^{5}$ Graefe ist bei seinem Verständnis des Textes gezwungen, die Pluralstriche zu ignorieren. 
und Kammerherrn der Gottesverehrerin Dj-Hnzw-snb, seine Mutter ist die Hausherrin und Dame $\breve{S} p-n$-3st, gerechtfertigt, Herrin der Ehrwürdigkeit: Mögest du den Ka seiner Väter ${ }^{59}$ in ihm dauern lassen: Das ist die Belohnung, die du ihm erweisen sollst; (denn) er ist ein Diener, der seinem Herrn folgt."

Die zentrale Bitte des Textes - denn $[m j] m$ htp $n[h m] . k$, falls denn so zu ergänzen ist, ist ja nicht mehr als eine Einleitungsfloskel - ist $\underline{d} d j . k k 3 n$ jtjw.f jm.f, und man fragt sich, was damit gemeint sein soll: Der „Ka der Väter“ ist ja aufgrund des anthropologischen Konzepts der Ägypter ohnehin in den Nachkommen erhalten ${ }^{60}$. Möglicherweise geht es hier jedoch um etwas ganz Konkretes: Der Bittsteller ist Schreiber der Gottesgemahlin, sein Vater und sein Großvater sind aber beide Schreiber und Kammerherrn (jmj-hnt). Das „Dauernlassen des Kas seiner Väter" könnte am Ende nichts anderes bedeuten, als daß auch er dieses Amt ${ }^{61}$ eines Kammerherrn erhalten möchte, das schon seine Väter innehatten. „Ka“ im Sinne von „Stellung, Rang“ ist im übrigen durchaus belegt ${ }^{62}$.

Graefes Übersetzung der einleitenden Hymne ist dagegen zuverlässig und auch sein Vorschlag, am Ende (Z. 8) wțz nhh $m$ [`wj.f] zu ergänzen, überzeugend. Ein Punkt dürfte aber zu korrigieren sein: In Z. 6-7 übersetzt er h3j.n.f $\operatorname{snw}(t) . f m-h t$ 迎 ligtum erhellte am Leibe der Ewigkeit, Sich-Verjüngender ...", aber W $_{1}$ kann außerhalb kryptographischer Texte $\mathrm{m}$. W. kaum $n h h$ gelesen werden. Es wird vielmehr ḩ3j.n.f $\operatorname{snw}(t) . f m h t-h h(w)$ rnpj zu trennen sein, also „... nachdem er sein Heiligtum erhellt hat im Leibe (der Himmelsgöttin), $H h$, der sich verjüngt ..." Zwar sind beim Sonnenlauf im allgemeinen die Rollen von Osiris (als Übergeber) und $H h$ (und $H h t$, als Empfänger) differenziert ${ }^{63}$, aber charakteristisch für diese Hymne ist es ja offenbar, daß Osiris am gesamten Kreislauf der Sonne beteiligt ist.

C. Vor einigen Jahren hat Ph. Derchain die Inschrift auf der Stehfigur des Astronomen, Astrologen, „Toxikologen“ und Magiers $H r$-3hbjt ausführlich kommentiert und und große Fortschritte im Verständnis dieser recht problematischen Inschrift erziel $t^{64}$. Zumindest eine Stelle in der oberen Hälfte von Z. 3 kann aber nicht richtig sein: Derchain übersetzt sie, im Anschluß an Neugebauer und Parker ${ }^{65}$, „(qui indentifie tout phénomène observé dans le ciel parce qu'il l'a attendu,) instruit de leurs conjonctions et de leurs phases". Diese selbe Stelle war früher von H. Brunner als Beleg für Windorakel gedeutet ${ }^{66}$ und durch „erfahren in ihren Winden und deren Wehen(?)“ übersetzt worden. Beide Übersetzungen sind indes nicht haltbar: Brunners Einwand gegen die Lesung von $\frac{}{\frac{2 "}{Y}}$ als $h n m$ und dessen Bedeutung „Konstellation, Konjunktion“ sind zweifellos

${ }^{59}$ Graefe übersetzt irrtümlicherweise „Ka deiner Väter“.

${ }^{60} \mathrm{Vgl}$. LÄ III, 275-6.

${ }^{61}$ Bzw. den Rang: nach Graefe, op. cit., Bd. 2, 88 ff., ist jmj-hnt ein Rangtitel.

${ }^{62} \mathrm{Vgl}$. Wb V, 89, 10 und meine Ägyptischen Biographien der 22. und 23. Dynastie, ÄUAT 8, 1985, 532, Z. 5 und $178(17)$

${ }^{63} \mathrm{Vgl}$. J. Assmann, Sonnenpriester, $40 \mathrm{ff}$.

${ }^{64} \mathrm{CdE}$ 64, 1989, 74-89. Seine Textwiedergabe ist allerdings etwas dadurch beeinträchtigt worden, daß er die Inschrift nicht selbst gesehen hat. So werden eine Reihe von offenkundigen Emendationen als tatsächliche Lesungen wiedergegeben - offenbar ein Irrtum bei der „Tradierung“ der Textkopie. Da ich den Text selbst kollationiert habe und auch Abdrücke der Inschrift habe, möchte ich betonen, daß die nicht wenigen Lesungen Derchains, die von Daressys Kopie abweichen, bis auf zwei Ausnahmen alle unberechtigt sind (die meisten Abweichungen berühren allerdings das Verständnis kaum). Diese beiden Ausnahmen stehen in Z. 2: Die Gruppe vor $\underline{d}$ d.f ist tatsächlich $\mathrm{O} \square$ zu lesen (und nicht $\odot /$ wie bei Daressy), und das letzte Zeichen vor der Lücke ist das Oberteil eines Vogels, der eher einem als einem (Daressy) ähnelt. Davon abgesehen ist Daressys Abschrift (soweit bei Drucktypen möglich) tadellos.

${ }^{65}$ Astronomical Texts, III, 215.

${ }^{66}$ In: Wort und Geschichte (Fs K. Elliger), AOAT 18, 1973, 27; 29 = H. Brunner, Das hörende Herz, OBO $80,1988,226 ; 228$. 


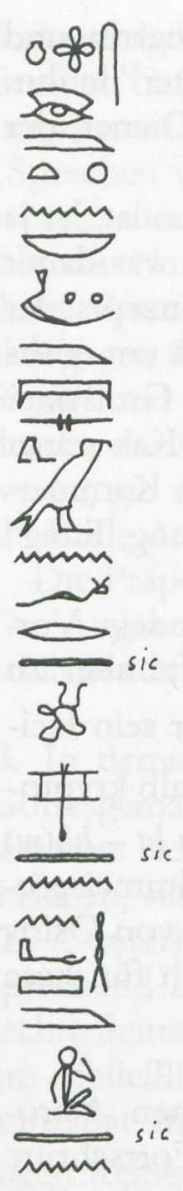

Abb. 1

berechtigt. Vor allem aber ist hinter hne nicht gsgs zu lesen, sondern deutlich $\longleftarrow$ (s. Abb. 1). Andrerseits ist aber auch Derchains Einwand gegen Brunners Übersetzung richtig: Sie paßt nicht in den Kontext, wo nur von der Beobachtung astronomischer Phänomene die Rede ist. Die richtige Lösung hatte schon H. de Meulenaere erkannt ${ }^{67}$ : Das vorhergehende Ajin ist noch mit dazuzuziehen, und dann ergibt sich `ร̌m, eine lautliche Schreibung für `hm „(ver)löschen“ (entspr. kopt. $\boldsymbol{\omega}(\boldsymbol{\omega} \overline{\mathbf{M}})$. Man muß nicht einmal eine Haplographie des $\_$von $h n^{\ulcorner}$annehmen, denn $h n$ ist ja in der Spätzeit eine ganz gängige Schreibung von $h n^{\ulcorner}$. Das mysteriöse 装..$s n^{68}$ sollte dann eine Art Gegenstück zu ${ }^{\complement} h \mathrm{hm}$ sein und das Sichtbarwerden der Himmelskörper bezeichnen. Eine (neben $t 3 w$ ) häufige Lesung des Zeichens ist $n f(w)$ u. ä. Von diesem Stamm gibt es ein Verb $n f j$,ausatmen, hauchen“ (Wb II, 250), das auch im Sinne von „(ein Feuer) anfachen" belegt ist" ${ }^{69}$, und diese Bedeutung könnte auch hier ganz gut passen: swn $m$ ht $n b \quad m 33 m$ pt z3w.n.f r.s šs $<m>$ $n f j . s n$ hne $n^{\top} m . s n$, der von allen Dingen weiß, die am Himmel zu sehen sind und auf die er gewartet hat, kundig (in) ihrem Anfachen (= Aufleuchten) und ihrem Verlöschen“. Dem läge also die Vorstellung zugrunde, daß das Licht der periodisch leuchtenden Himmelskörper ein Feuer ist, das angefacht wird und dann wieder verlöscht.

D. Zu den Inschriften der schon mehrfach behandelten Statuenbasis London BM 512 des P3-dj-B3stt $t^{70}$ aus spätptolemäischer Zeit gehört neben dem bekannten „Festkalender des Imhotep “71 auf einer der Längsseiten (vermutlich der Vorderseite) auch eine Darstellung seiner Frau, gefolgt von drei Töchtern ${ }^{72}$. Die Töchter werden nur jeweils durch (Titel,) Namen und Filiation identifiziert. Der Ehefrau wird zusätzlich noch eine kurze Rede in den Mund gelegt (s. Abb. 2), und dieser kurze Text ist in den Bearbeitungen von Gauthier und Wildung nicht richtig verstanden worden. Gauthier ${ }^{73}$ liest ihn als ein Gebet mit einem einleitenden Satz: „Sa grande femme ... Elle dit toutes ses demandes à Hathor ... écoutant les prières: ,Donne-moi la faveur d'[avoir?] un fonctionnaire(?) ... Que tout ce qu'il dit en réponse soit bon! Donne-moi la faveur d'être aimée de lui et de [mes] enfants!" "Das kann aber so nicht stimmen: Es steht gar nicht „toutes ses demandes“ da, sondern nur $n h w w n b$, und das ergäbe in einem einleitenden Satz, wie ihn sich Gauthier vorstellt, wenig Sinn (vielmehr würde man dann so etwas wie *,Sie sagt als [ihre] Bitte zu Hathor" erwarten). Zudem faßt Gauthier gleich zweimal ein „donne-moi“ auf, aber dann wäre *jmj n.j zu erwarten. Auch das im letzten Satz an dj $n . j$ anschließende hzwt mrwt $m$ - hr.f kann kaum als „la faveur d'être aimée de lui" verstanden werden.

Wildung $^{7{ }^{\circ}}$ versteht den Text anders, und seine Auffassung ist der Gauthiers deutlich überlegen: „Seine große geliebte Gemahlin ... sie sagt alle Bitten zu Hathor ... die die Gebete erhört, die mir gab das Geschenk eines großen Fürsten ... dessen Antworten allesamt zum Besten dienen,

\footnotetext{
${ }^{67}$ Bei Neugebauer-Parker, op. cit., 215.

${ }^{68} \leftrightharpoons$ ist natürlich $\rightarrow-$ zu lesen, wie mehrfach in dieser Inschrift. Vermutlich hat der Steinmetz einfach die beiden Ausbuchtungen in der Mitte vergessen.

${ }_{69}$ S. Meeks, Année lexicographique, 79.1526; G. T. Martin, The Tomb of Hetepka, EES, Texts from Excavations, 4, 1979, 11; pl. XII (13).

${ }^{70}$ H. Gauthier, BIFAO 14, 1918, 33-49; pl. 1; D. Wildung, Imhotep und Amenhotep, MÄS 36, 1977, 73-8 (\$ 47); Taf. XIV-XV.

${ }_{11}$ S. dazu G. Vittmann, „Bemerkungen zum Festkalender des Imhotep“, Fs Westendorf, 947-61.

${ }^{72}$ Wildung, op. cit., Taf. XV.

${ }^{73}$ Op. cit., 36.

${ }^{74}$ Op. cit., 74.
} 


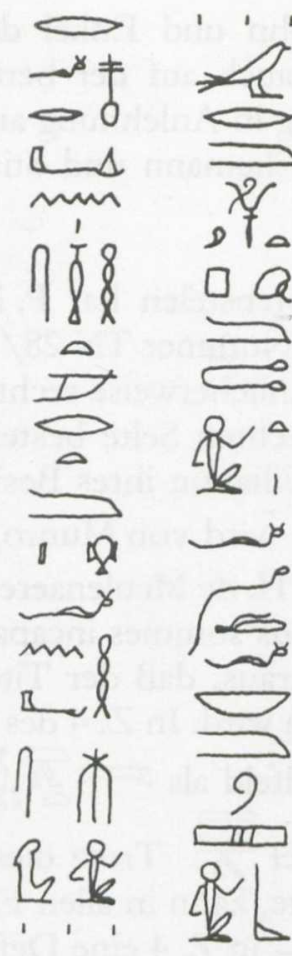

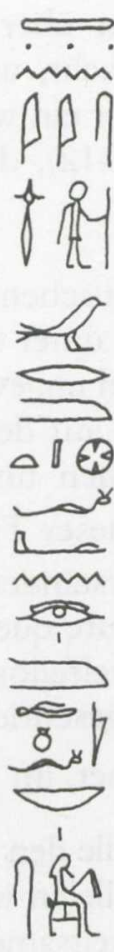

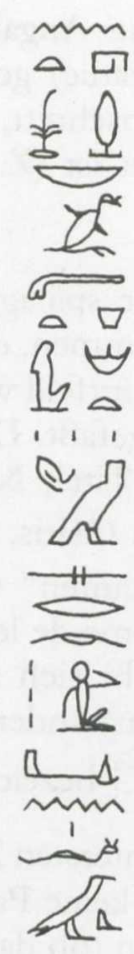

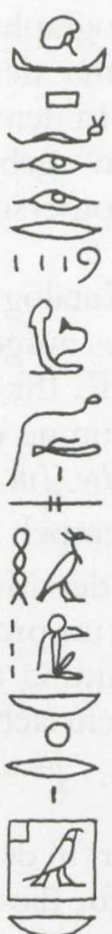

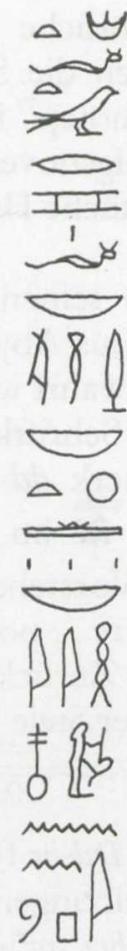

Abb. 2

der mir Gunst und Beliebtheit vor seinem Angesicht und (viele) Kinder schenkte. “ Diese Übersetzung ist grammatisch unanfechtbar, aber sie hat ein anderes Manko: Bei ihr wechselt der Text mitten im Satz von der 3. in die 1. Person und entsprechend von einem deskriptiven Zusatz zum Namen (,sie sagt") in einen biographischen Redestil („,die mir gab“). Will man das vermeiden, muß der ganze Text etwas anders zu verstehen sein. Sowohl bei Gauthier wie auch bei Wildungs Übersetzung wird das auf den Namen folgende $\underline{d} d . s$ in den folgenden Text miteinbezogen, und das ist alles andere als naheliegend, denn üblicherweise bilden ja (Titel +$)$ Namen $+\underline{d} d$.fld dd.s nur die Einleitung, und erst danach beginnt die eigentliche Rede. Auch hier ist zweifellos so zu gliedern, und damit ergibt sich kein Verbalsatz, sondern ein Adverbialsatz mit dem Prädikat $\operatorname{dj}(w)(n . j)$ : „Alles, was (ich) von Hathor erbeten habe ${ }^{75} \ldots$ ist mir gewährt worden "; das folgende $f q 3 w$ ist dann eine Apposition zu $n h w(. j$ ?) $n b$. Insgesamt lautet die Rede nun: „Alles, was (ich) von Hathor erbeten habe, der Herrin der südlichen Sykomore, der Herrin der Männer und Gebieterin der Frauen, die die Bitten erhört, ist mir gewährt worden, (nämlich) das Geschenk eines hohen Beamten, groß in seiner Stadt, gut in seinem Charakter, ein Herr von Reichtümern, bedeutend in seinem Amt, der Erste unter seinen Kollegen ${ }^{76}$, - alles, was er sagt, bedeutet ein Antworten in guter Weise ${ }^{77}$. Mir sind Gunst und Liebe bei ihm geschenkt worden sowie Kinder.“

Die Gliederung dieses kurzen Textes ist sicher etwas ungewöhnlich, zweifellos deshalb, weil in eine kurze biographische Aussage der Ehefrau eine Reihe von Epitheta ihres Mannes, des Besitzers der Statue, eingebaut worden ist. Vergleichbares ist in späten Biographien auch sonst belegt: Auf der Stele Wien 5857 z. B., in der Biographie der Tathotis ${ }^{78}$, werden in Z. 4-5 recht

${ }^{75}$ Ebensogut möglich wäre ein Partizip: ,alles von Hathor Erbetene“. Unter dem $h r$ steht ein überflüssiger Füllstrich, ebenso unter $\underline{d} d$.

${ }^{76}$ Zu tt s. Gardiner, JEA 24, 1938, 170-1; 179.

${ }^{77}$ Mit dieser ungewöhnlichen Formulierung könnte gemeint sein, daß er in seinem Reden auf die Bedürfnisse und Empfindungen seiner Gesprächspartner eingeht, darauf ,antwortet“.

${ }^{78}$ G. Vittmann, SAK 22, 1995, 283-323. 


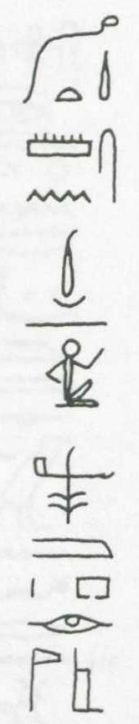

毕

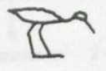

m

90

舟
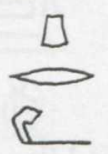

10
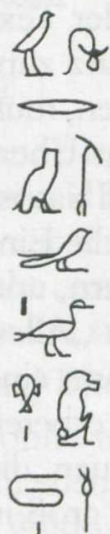

?!

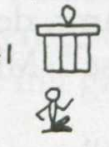

ausführliche biographische Angaben über Sohn und Enkel der Tathotis (vermutlich die Stifter der Statue) gemacht, und auch auf der berühmten Stele der Taimhotep ${ }^{79}$ ist in dem Abschnitt, der ein wenig in Anlehnung an den Texttyp der „Königsnovellen“ gebildet ist (Z. 8-12), der Ehemann und Stifter der Stele die eigentliche Hauptperson.

E. In seinen Katalog der spätägyptischen Totenstelen hat P. Munro auch eine Stele aus Abydos aufgenommen, die unter der Nummer TN 28/10/24/2 in Kairo aufbewahrt wird ${ }^{80}$. Ihr Hauptfeld wird ungewöhnlicherweise rechts und links von je einer Schriftkolumne eingefaßt. Die auf der rechten Seite besteht nur aus einem Vermerk $d d-m d w(j) n+$ Titel, Namen und Filiation ihres Besitzers ` $n h-W n-n f r$, eines im Tempel des Osiris. Dieser Titel wird von Munro, sicher zu Recht, als „Vorsteher der Musikanten“ verstanden ${ }^{81}$. H. de Meulenaere meint dagegen ${ }^{82}$, „le titre ... pose un problème de lecture que nous sommes incapable de résoudre". Seine Zurückhaltung ergibt sich zweifellos daraus, daß der Titel des ' $n h-W n-n f r$ auf der Stele mehrfach ganz anders geschrieben wird: In Z. 4 des Haupttextes wird

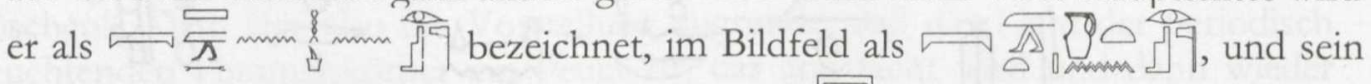
Sohn $\underline{D} d-\underline{h r}$ führt in der untersten Zeile den Titel $\overparen{\pi}$. Trotz dieser merkwürdigen Schreibungen, für die ich keine Parallelen wüßte, kann in allen Fällen aber nur der Titel $h r j s m^{\top} w$ gemeint sein (ob das seltsame $\_$in Z. 4 eine Deformation aus ist, läßt sich schwer entscheiden). In zwei Fällen hat der Titel allerdings noch einen Zusatz, der gleichfalls schwer verständlich ist. In Z. 4 dürfte am ehesten $n h b$, des Festes“ (in einer Art „alphabetischer Schreibung") gemeint sein, also „Leiter der Festmusik des Osiris“, im Bildfeld vielleicht $n w d h w$,des Opfertisches (des Osiris) “ o. ä. Gemeint ist mit diesen Zusätzen womöglich der Unterschied der Musik bei Tempelfesten und beim gewöhnlichen Kult.

$\mathrm{Daß}$ auf jeden Fall der Haupttitel von Vater und Sohn hrj $\varsigma m^{\ulcorner} w$ ist, wird auch durch die Inschrift der linken Randzeile bestätigt: Der Text dieser Kolumne (s. Abb. 3) wird von Munro in sehr entstellter Form wiedergegeben und offenbar als Titulatur mit Namen und Filiation verstanden. Tatsächlich hatte aber schon Mariette $^{83}$ die Inschrift in völlig korrekter Form wiedergegeben, soweit das nach dem Foto bei Munro zu beurteilen ist ${ }^{84}$. Es handelt sich um einen kurzen biographischen Text mit ziemlich ungewöhnlichem Inhalt: Die musikalische Tradition im Tempel wird wiederaufgenommen, nachdem sie, offenbar für längere Zeit, erloschen war ${ }^{85}$ : „Sprechen von Worten: ,Ich habe die Musik (bzw. den Gesang) im Tempel des Osiris wieder eingerichtet, als sie zuvor zugrundegegangen gefunden

Abb. 3

${ }^{79}$ London BM 147, s. E. A. E. Reymond, From the Records of a Priestly Family from Memphis, ÄA 38, 1981, $165-77$.

${ }^{80}$ P. Munro, Die spätägyptischen Totenstelen, ÄF 25, 1973, 304; Taf. 44; vgl. auch A. Mariette, Catalogue général des monuments d'Abydos, Paris 1880, 489 (1296).

${ }^{81}$ Vgl. dazu Wb IV, 479, 4-5; Meeks, Année lexicographique, 78.4120.

${ }^{82}$ OLP 6/7, 1975/76, 149-50.

${ }^{84}$ Op. cit., Taf. 44. Lediglich der Ideogrammstrich neben dem 占 ganz unten läßt sich auf dem Foto nicht klar erkennen, er könnte aber durchaus vorhanden sein.

${ }^{85}$ De Meulenaere (OLP 6/7, 1975/76, 150 oben) hält die Inschrift dagegen für einen biographischen Bautext 
worden war. Mein Sohn $\underline{D} d$ - $h r$ wird mein Nachfolger sein" ${ }^{\text {(86. }}$. Die Angabe, daß er den wiedereingerichtet habe und und sein Sohn ihm darin folgte, paßt exakt zum Titel hrj šm`w, den ja Vater und Sohn tragen, und bestätigt so die Lesung des Titels.

Neben der Schreibung des Titels (wo zumindest in zwei Fällen das ${ }^{\top}$ von $s m^{\top} w$ nicht erscheint) enthält die Stele eine weitere Merkwürdigkeit, und interessanterweise wird dort ein Ajin zuviel gesetzt: Sowohl der Vater als auch der Sohn des Stelenbesitzers heißen $\underline{D} d$ - $h r$; der Name des Vaters wird einmal (rechte Rahmenzeile)

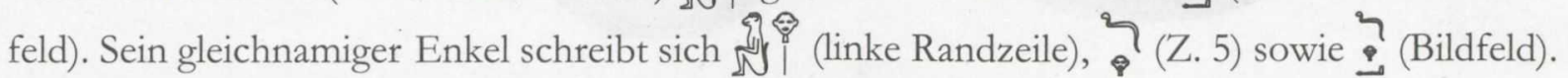
Beides scheint auf eine Entwertung oder Schwächung von (auslautendem) Ajin zu deuten. Demnach würde man die Datierung der Stele durch Munro (ca. 300-250 v. Chr. bzw. „sebennytisch-frühptolemäisch“) gerne etwas nach unten verschieben, während umgekehrt eine Schreibung wie 8 für „Fest“ (falls so zu lesen ist) eher auf eine etwas frühere Zeit, etwa die 30. Dynastie, deuten könnte. Allerdings können ,alphabetische“ Schreibungen durchaus auch noch in ptolemäischer Zeit vorkommen, vgl. etwa $4 \int$ als $j b$ „Herz“ in dem oben (Teil I) unter A, 7 aufgeführten Beleg.

(, la construction annexe du ,domaine d'Osiris‘ que le propriétaire de la stèle se vante d'avoir réédifié porte un nom tout aussi mystérieux"), sicher aufgrund der gerade in Bauinschriften so häufigen Phrase gmj.n.tw.f ... w3j $r$ w3sj.

$\left.{ }^{86}{ }_{z 3} 3 . j\right) D d-h r\langle r\rangle w d b s 3 . j$. Denkbar wäre natürlich auch $\langle h r\rangle w d b s 3 . j$ und die Unterordnung der Phrase als Umstandssatz: ,,indem mein Sohn $\underline{D} d-\underline{h r}$ dabei ist, mir nachzưfolgen“ (wenn etwa die Stele unmittelbar nach dem Tod des Vaters errichtet worden war). 